\title{
Singularité du Bois Borassus Aéthiopum par sa physiologie
}

\author{
Komlan Lolo, (doctorant en Sciences et Technologies) \\ Sonnou Tiem (Enseignant-Chercheur)
}

Université de Lomé, ENSI, Laboratoire Génie Mécanique, BP 1515 Lomé

Doi: 10.19044/esj.2017.v13n33p463 URL:http://dx.doi.org/10.19044/esj.2017.v13n33p463

\begin{abstract}
Among the traditional building materials in Togo, the Borassus Aéthiopum or Rônier is one of the most widely used. The singularity of this wood made it famous because of its resistance to xylophages and to the various inclement weather. But the determining factors of this resistance are unknown. This work aims to show the singularity of this wood under different observation angles. In this work, we study the physiology of this wood. We will observe samples taken at different locations under different scales. In view of the results obtained, we decide on the particularity of this wood. The rônier is made of detachable fibers.
\end{abstract}

Keywords: Rônier, fibers, development, growth.

\section{Resume}

Parmi les matériaux de construction traditionnelle au Togo, le Borassus Aéthiopum ou Rônier fait parti des plus utilisés. La singularité de ce bois l'a rendu célèbre de par sa résistance aux xylophages et aux diverses intempéries. Mais les facteurs déterminants de cette résistance sont méconnus. Ce travail vise à montrer la singularité de ce bois sous des angles différents d'observation. Dans ce travail, nous étudions la physiologie de ce bois. Nous observerons des échantillons pris à des endroits différents sous de différentes échelles. Au vu des résultats obtenus, nous nous prononçons sur la particularité de ce bois. Le rônier est formé de fibres détachables.

Mots clés : Rônier, fibres, développement, croissance.

\section{Introduction}

Depuis l'aube des temps, le bois a toujours servi de matériau de construction dans plusieurs domaines. Malgré, l'apparition de nouveaux matériaux, le bois a toujours gardé sa noblesse dans son utilisation à travers le monde. Dans le cas du Borassus dont le bois présente une singularité par 
sa résistance mécanique et surtout par ses propriétés spécifiques, nous avons constaté que ce bois utilisé dans les constructions des ouvrages reste intact après de nombreuses années. Il ne se déforme pas, et n'est pas attaqué par les moisissures d'où l'intérêt d'étudier la physiologie de ce bois.

Le bois provient de la partie dure d'un arbre. Un arbre est une plante terrestre ligneuse comportant un tronc, les branches, les feuilles et les racines. Toutes ces parties sont formées de tissus végétaux. Le bois est utilisé dans presque tous les domaines. Suivant la nécessité, on effectue une étude sur le développement et la croissance de l'arbre. L'arbre se développe du cœur (milieu) vers l'écorce (extérieur) avec une juxtaposition des couches formées de cellules. La formation est faite de manière concentrique en couche et par an. Chaque couche annuelle est appelée cerne. Elle permet de déterminer l'âge de l'arbre. La forme du tronc d'un arbre est pour la plupart conique (Hazard et al., 2013).

Le Borassus est un bois tropical qu'on rencontre en Afrique, au sud de l'Asie en Inde pacifique. Il appartient à la famille des palméa. Il existe deux types : une espèce ventrue rencontrée en Afrique et une autre rencontrée en Asie. L'espèce ventrue d'Afrique est Aéthiopum. Parmi les espèces, nous avons un sujet mâle et un sujet femelle. Le sujet mâle ne porte pas de fruits mais celui femelle porte des fruits (Giffard, 1967). La morphologie du Borassus ne respecte pas exactement ce qu'on trouve chez un bois ordinaire. Il est assimilable au bois. Son tronc est appelé faux tronc en botanique mais sa forme est plus régulièrement cylindrique que chez un bois ordinaire. Il se développe plutôt des racines vers le sommet tout en gardant l'assise cellulaire de base. Le Borassus ne possède pas de branches donc ne possède pas de nœud. Son développement n'a pas l'activité du cambium (croissance suivant le rayon). Avec l'absence de la formation des cernes, il est difficile d'apprécier l'âge de l'arbre (Giffard, 1967). La technique de détermination de l'âge n'est pas connue. La structure du borassus est très fibreuse et détachable. Les fibres sont liées par une matrice à dominance variable. Les fibres sont distinctes de la matrice. C'est un matériau innovant à découvrir.

Quelques études (très peu) sont faites sur l'espèce d'Afrique : influence des changements climatiques sur sa distribution géographique, distribution géographique, aspect technico-économique de la transformation au Bénin (Gbesso et al., 2014). La caractérisation du borassus physique et mécanique du Borassus est faite au Bénin sur leurs essences. Au Togo, le rônier ou Borassus Aethiopum a fait objet de recherche par la détermination des caractéristiques mécaniques et la tenue au feu. Giffard a présenté la forme cylindrique du tronc avec son phénomène de desquamation. Il présente l'utilité faite des pétioles, des feuilles et de fruits. Le climat, l'influence de l'homme et le sol sont des facteurs qui influencent le 
développement de l'arbre. Les études faites par les auteurs précédents sur le borassus n'ont pas mentionné la morphologie issue du bois issu de cet arbre. La morphologie n'est pas débattue. Parmi les maigres études, aucune n'a présenté la formation et le développement du tronc. Sa différence, dans son développement, n'est jamais ressortie. La structure et la texture des fibres de cet arbre n'ont jamais été présentées.

Dans ce travail, nous présenterons la structure et la texture du Borassus Aéthiopum sous trois angles d'observations. Du point de vue macroscopique, microscopique et nanoscopique, la structure du borassus est présentée et ensuite nous discuterons en ressortant la particularité de ce bois. La conclusion de ce travail ouvrira la piste à d'autres études sur ce bois.

\section{Matériels et méthodes}

Dans cet article, nous avons étudié le bois Borassus Aéthiopum. Le Borassus est un arbre de la famille des palmiers. Le développement des palmiers est différent de celui d'un arbre ordinaire. L'arbre a la forme d'une colonne cylindrique de 30 à 40 centimètres de diamètre et pouvant atteindre une hauteur de 20 mètres. Pour avoir une idée de l'arbre, nous présentons un jeune plant de quinze ans à la figure 1 .

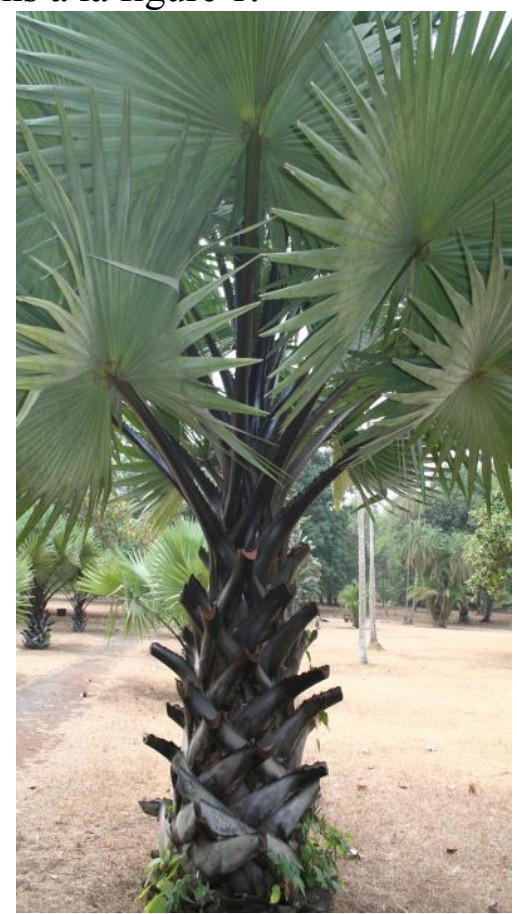

Figure 1 : Jeune plant de Borassus Aéthiopum

Nous avons observé un tronc d'un arbre abattu sur lequel nous apercevons visiblement les fibres. Les fibres sont donc des gaines longues 
dures de couleurs noires. Nous pouvons les détacher les unes des autres. A partir d'un autre borassus de même texture, nous prélevons des éprouvettes que nous observons au microscope optique. Des échantillons sont pris sur un autre tronc de même texture.

Le microscope donne une image agrandie d'un objet. Il est un outil qui permet d'avoir une certitude sur l'identification, les caractéristiques de la structure ainsi que sur la dimension de membranes. Six (06) morceaux de Borassus ont été découpés en forme prismatique de dimensions $15 \times 20$ × 30 $\mathrm{mm}^{3}$. Ils sont pris dans la périphérie du tronc, proche de l'écorce, dans le sens longitudinal. Toutes les faces sont usinées. Ces morceaux sont polis à la machine de marque Struers et au papier verre de 220 pouces fait en carbure de silicium. Au cours du polissage, l'eau est utilisée pour le refroidissement et l'évacuation des copeaux. Les échantillons polis sont de nouveau séchés à environ dix-huit degré $\left(18^{\circ}\right)$ Celsius pendant vingt-quatre (24) heures. Après le polissage, les échantillons sont alors destinés à la visualisation. Afin de bien pouvoir présenter le plan d'observation aux lentilles, l'échantillon est posé sur une gomme, puis pressé au moyen d'un vérin de pressage. L'ensemble est monté sous la lentille du microscope (figure 2).

Pour un échantillon, nous observons trois points sur la face. A chaque point, nous varions les miroirs. Pour avoir une bonne netteté, les miroirs ont été variés suivant des grossissements : $500 \mu \mathrm{m}, 200 \mu \mathrm{m}, 100 \mu \mathrm{m}$ et $50 \mu \mathrm{m}$. Nous présentons uniquement ici la coupe longitudinale et transversale, vue au $500 \mu \mathrm{m}$, au $200 \mu \mathrm{m}$ de deux endroits et $100 \mu \mathrm{m}$. Les informations sur les clichés témoignent du phénomène d'agrandissement. La figure (4) montre la structure dans le plan longitudinal. La figure (5) montre la structure dans le plan transversal.

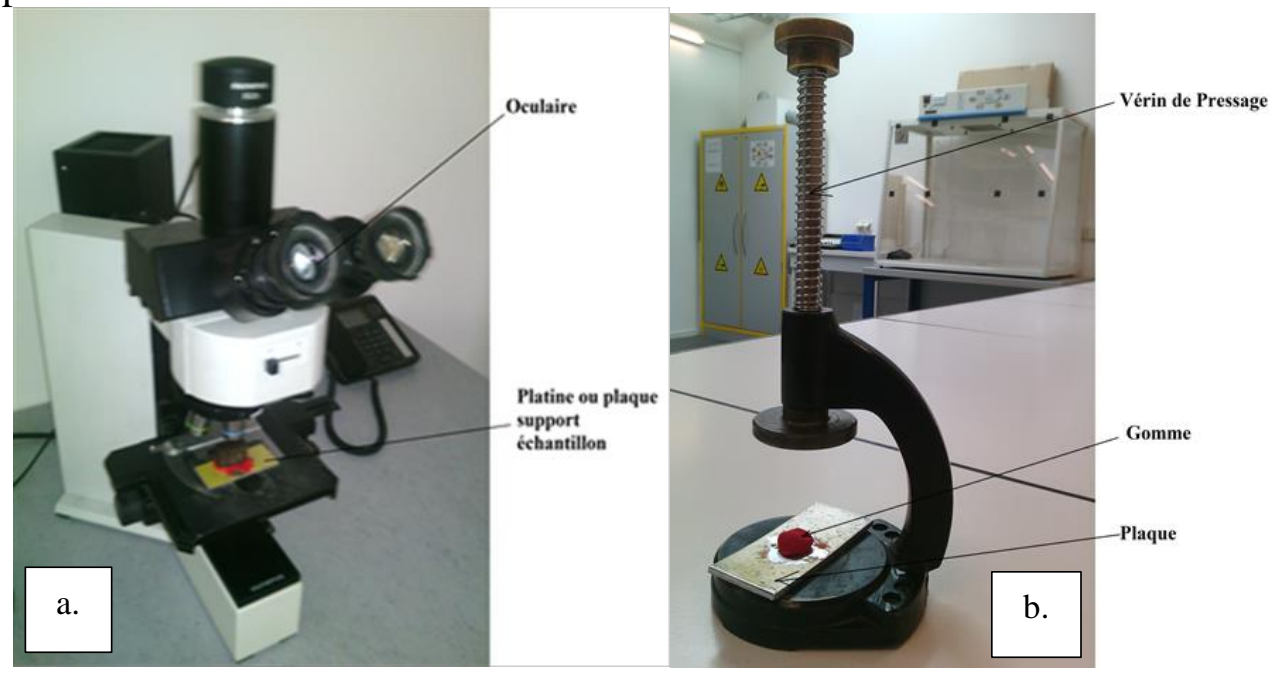

Figure 2 : Microscope avec le dispositif de planéité a. Microscope ; b. Dispositif de planéité 


\section{Résultats et discussions}

La figure 3 nous montre l'observation macroscopique. L'observation à l'œil nu fait remarquer la disposition des fibres. Les fibres se sont entrelacées dans un corps blanchâtre. Elles sont noires et sont entourées d'une substance qui les retiennent entre eux : c'est la colle ou liant. Les liants enrobent les fibres et assurent la liaison entre les fibres. L'ensemble est similaire à un matériau composite où nous avons les renforts qui baignent dans une matrice.

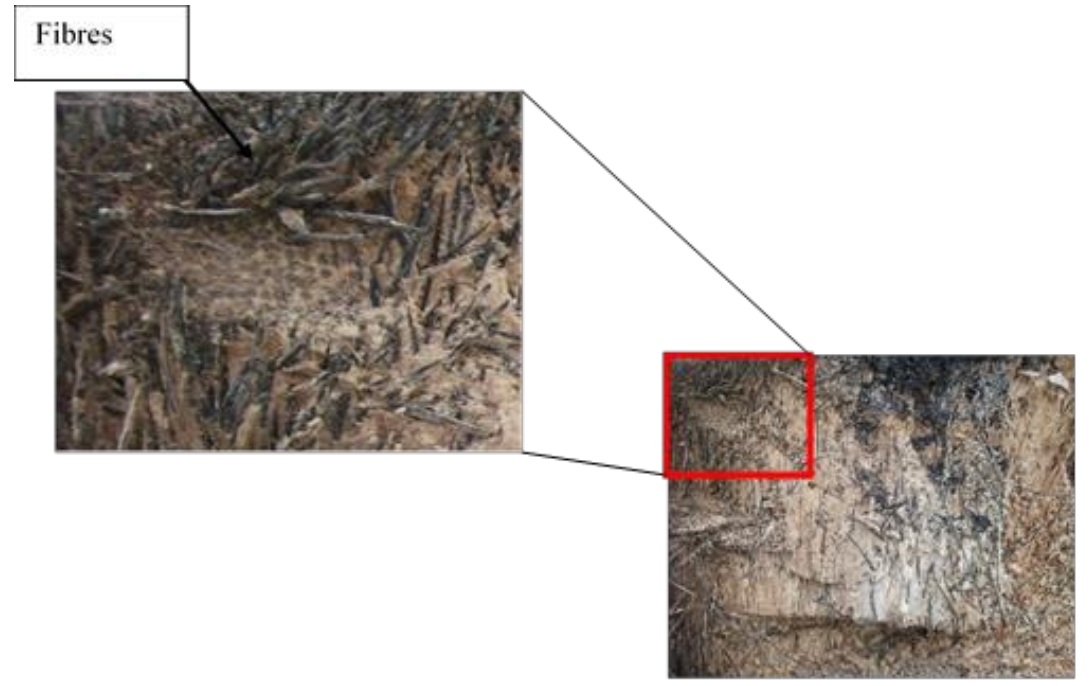

Figure 3 : Observation macroscopique du tronc

L'observation au microscope permet de tirer des conclusions sur la présence ou non de certaines anomalies et de juger de leur gravité. L'observation au microscope présente des avantages divers selon la technique de microscopie et le type de microscopes choisis. Au microscope, l'observation à $500 \mu \mathrm{m}$ (le plus petit agrandissement) des différents échantillons fait remarquer la présence des taches noires. Nous appelons ces taches noires les fibres. Entre les fibres, nous notons la présence d'un liant.

La figure 4 est la vue de l'échantillon dans le plan transversal. Suivant ce plan transversal, l'observation au microscope révèle que les fibres sont formées des petites fibres. Les fibres sont des torons ou regroupements des fibrilles. Elles sont entourées de liants. Le rôle des liants est de lier, entre elles, les fibres. Les fibrilles sont produites par la fibre souche. Elles sont alors des tissus fondamentaux de soutien. Ces fibrilles sont intrinsèquement liées. Elles n'ont aucune matière d'enrobage. Elles ont une forme polygonale Une fibre est produite et se développe de manière progressive depuis les racines jusqu'à la feuille. Le développement longitudinal des fibrilles est le développement en hauteur des fibres. Les fibrilles ont une forme hexagonale régulière mais sont de dimensions variées. 


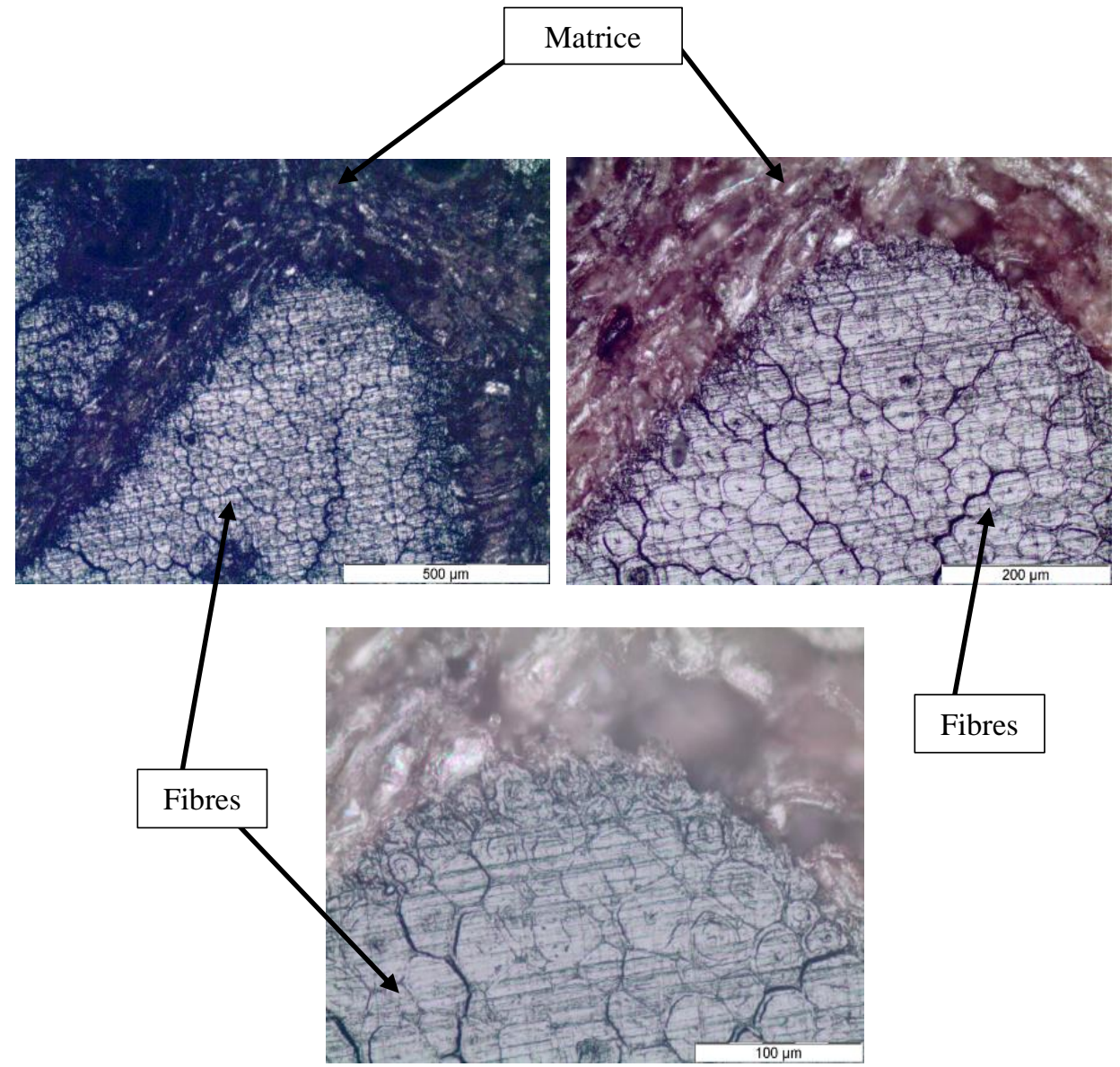

Figure 4 : Fibre entourée de liant dans le plan transversal

Suivant le plan longitudinal, les fibres se développent en formant des hexagones allongées (figure 5). C'est donc une forme particulière de développement des fibres. Le développement des fibres dépend de celui des fibrilles. Chacune des fibrilles s'allonge tout en gardant une forme donnée. Elles se développent dans le sens de la longueur. C'est aussi une autre forme de développement. 


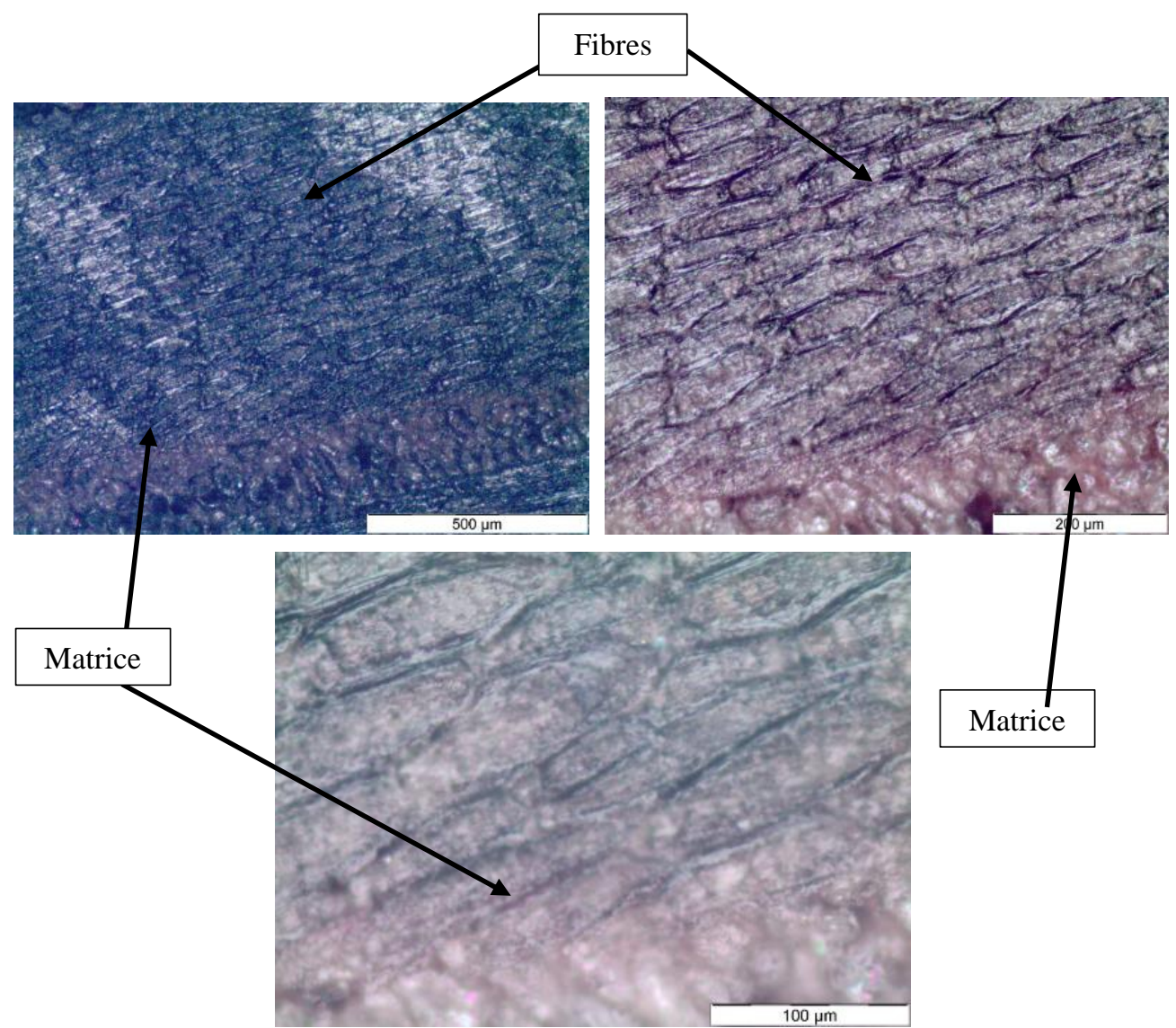

Figure 5 : Développement des fibrilles dans la direction longitudinale-plan tangential

Le microscope permet d'observer le développement longitudinal des fibres par la croissance des fibrilles. Ceci demeure une particularité à ce bois. Cela s'explique mieux sur la figure suivante 6. L'arbre ordinaire a l'axe noté longitudinale (L) comme axe privilégié de croissance. Il se développe plus dans cette direction en s'élargissant dans la direction radiale (R) par contre, l'arbre Borassus se développe dans la direction radiale (R) en formant d'abord son tronc avant de s'élever. Les fibres sont aussi formées avec les fibrilles. Les fibres ne grossissent plus mais plutôt se développent. La croissance et le développement du bois ordinaire ne ressemblent pas à cette description. 

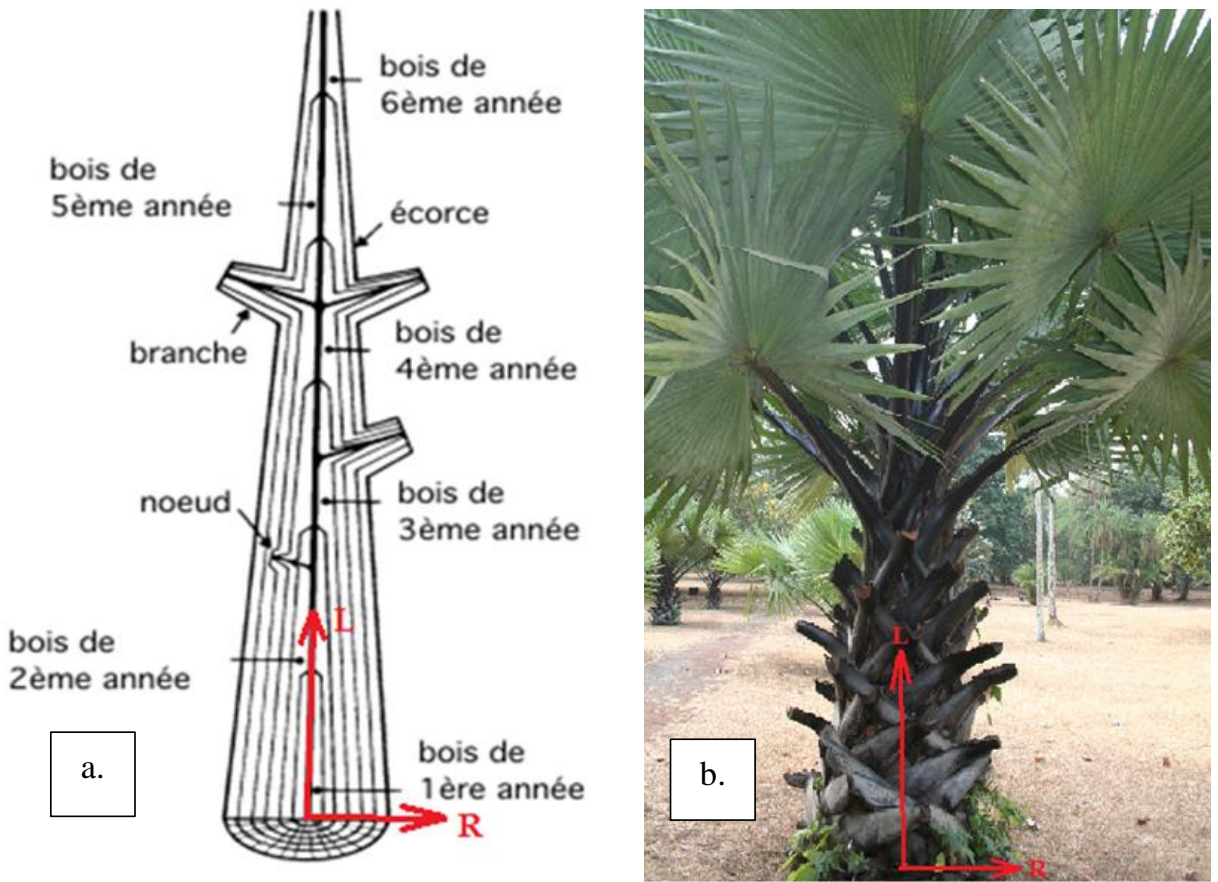

Figure 6 : Croissance et développement de deux espèces d'arbre

Arbre ordinaire b. Arbre Borassus

Le microscope ne donne pas des informations sur la structure interne du matériau. Ceci nous a conduits à solliciter une méthode d'observation plus performante qui est la tomographie.

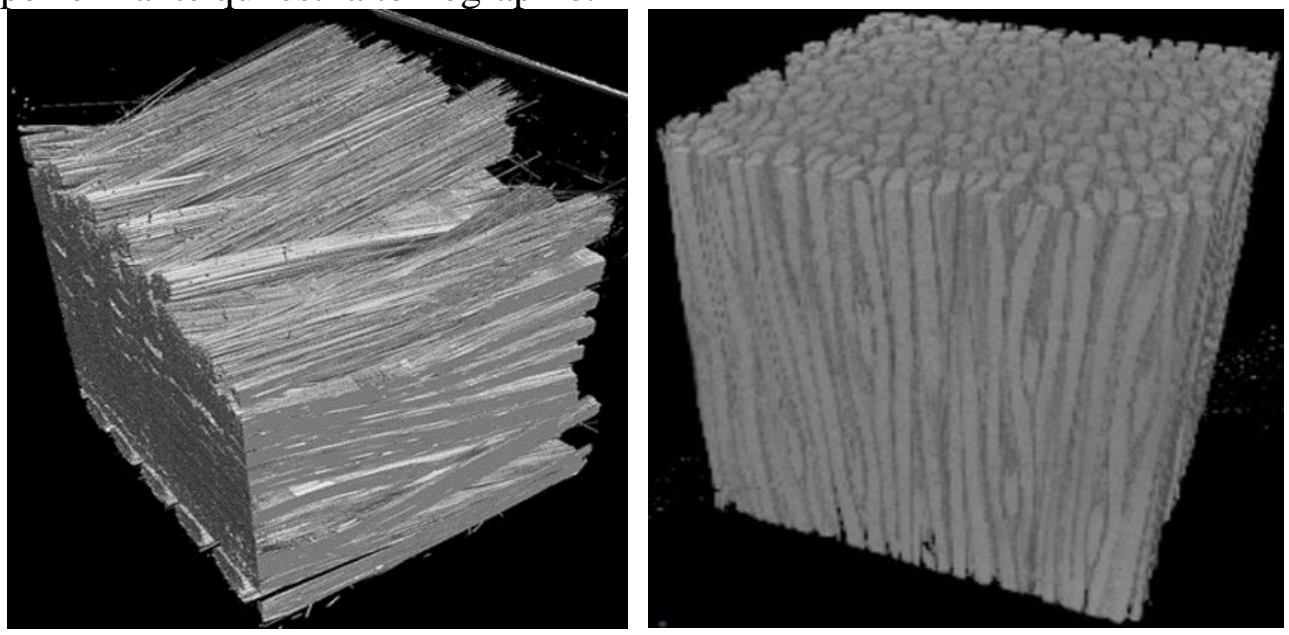

Figure 7 : Morceau de bois Borassus tomographié

La tomographie du morceau du bois borassus révèle l'entrelacement des fibres (figure 7). Elle montre aussi la présence des fibrilles regroupées en fibres. Nous voyons bien les fibres et les fibrilles. Les fibrilles constituent les 
nouvelles couches qui se forment par sa saison ou par an chez un bois ordinaire. Mais chez le Borassus, elles ne se multiplient pas, plutôt leur tailles sont assujetties à une croissance vers la cime de l'arbre. C'est pourquoi nous disons que le développement du borassus ne suit pas les mêmes principes qu'un bois ordinaire. Il ne se développe pas du cœur vers l'écorce mais des racines vers le haut. Le développement est plus accentué dans les directions radiale-tangentielle que axiale. Dans les premières années, le Borassus se développe plus dans la direction radiale jusqu'au diamètre final avant de croître en hauteur. L'arbre est nourri directement des fibres formées. Les fibres sont reliées aux racines et aux palmes. Les fibres sont les éléments de soutien comme d'ordinaire à l'arbre. A partir de la figure 8, nous voyons les formes des fibres suivant les trois directions considérées sur un arbre.
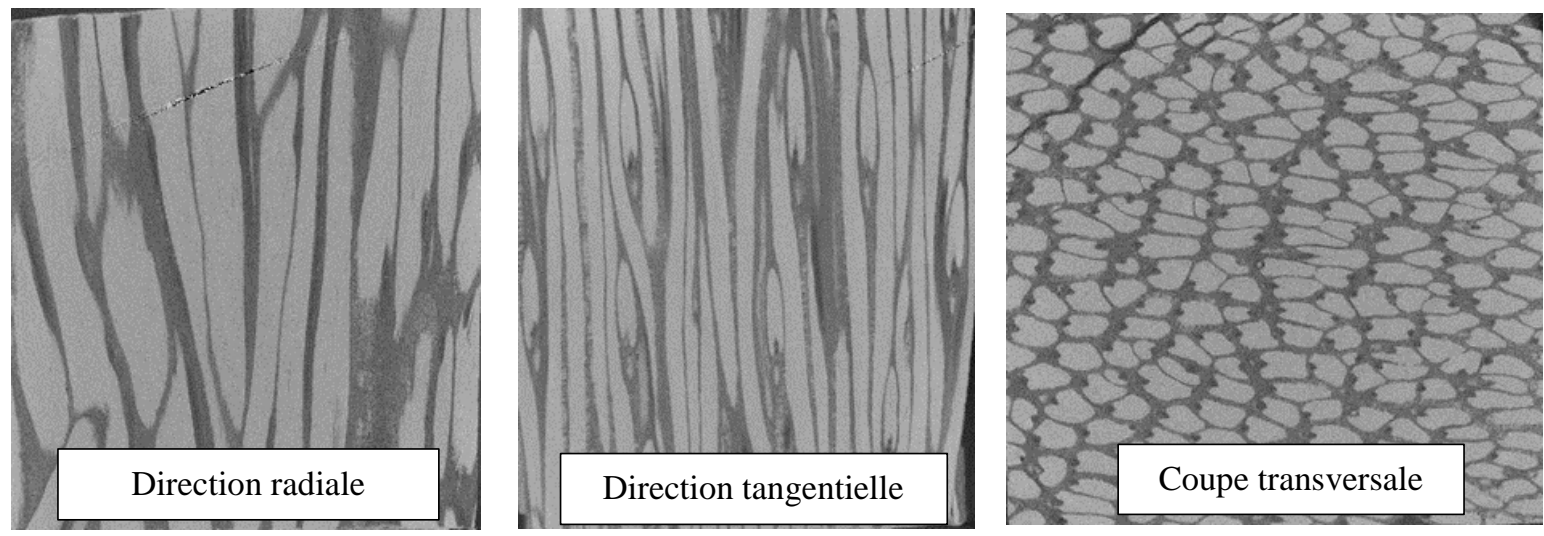

Figure 8 : Formes des fibres suivant les trois directions

La figure 8 est l'image du bois Borassus pris dans la zone dure près de l'écorce. Il y a moins de liants mais beaucoup de fibres. Les différentes figures (2 et 8 ) confirment les images présentées (figure 9) par P. Corbineau sur les bois. L'auteur a présenté quelques essences parmi lesquels se trouve le Borassus.
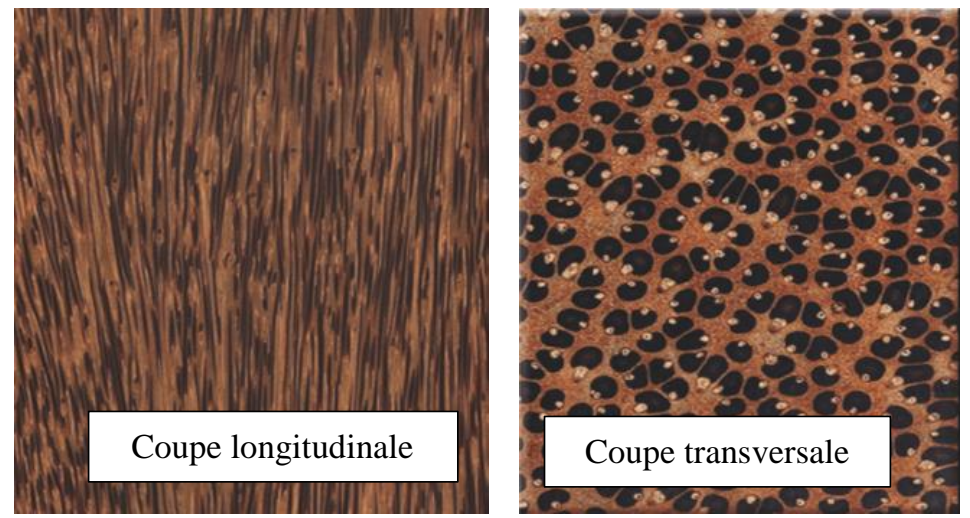

Figure 9: Images du bois Borassus selon P. Corbineau (P. Corbineau et al., 2009) 
Les images présentées s'apparentent à celles que nous avons obtenues à l'échelle macroscopique et à l'échelle nanoscopique. Les taches noires sont les fibres. L'analyse de l'image montre que l'échantillon a été prélevé dans la zone intermédiaire du bois c'est-à-dire pas au niveau de l'écorce ni au milieu du tronc.

\section{Conclusion}

Le bois Borassus est un matériau de construction très fiable, avec une absence de porosité au niveau de l'écorce. Son caractère fibreux confirme son appartenance aux matériaux nobles. L'étude de la structure prouve la différence de sa structure avec celle du bois ordinaire. Les fibres sont les regroupements de fibrilles en forme de torons de fibrilles. Cette forme de structure est un atout du point de vue mécanique qu'il faut étudier. La connaissance de son comportement mécanique justifiera ses performances dans les constructions de BTP (Bâtiments et Travaux Publics).

\section{References:}

1. Corbineau, P., \& Flandin, J.-M. (2009). Identification des bois. Esthétique et singularités (Vial.)

2. Gbesso, F., Yedomonhan, H., Tente, B., \& Akoegninou, A. (2014). Distribution géographique des populations de rôniers (Borassus aethiopum Mart, Arecaceae) et caractérisation phytoécologique de leurs habitats dans la zone soudano-guinéenne du Benin. Journal of Applied Biosciences Journal, 743.

3. Giffard, L. P. (1967). Le rônier. Revues Bois et Forêts des Tropiques, $116,1-54$.

4. Hazard, C., \& Mayer, J. (2013). Bois et matériaux associés (Delagrave., p. 515).

5. Kremer, A. \& Roussel G. (1982). Composantes de la croissance en hauteur chez le pin maritime (Pinus pinaster Ait.). Ann. For. Sci, p $15-34$. 